\title{
LES LECTEURS PARLENT
}

\section{A propos d'une formule}

L'article de M. l'Ingénieur Principal des Eaux et Forêts Koebelé à Sélestat, paru dans le numéro d'avril sur les indemnités dues aux propriétaires forestiers dépossédés, doit retenir toute l'attention. Pour ma part, il m'a vivement intéressé, car il a trait à un sujet d'estimation forestière 《erga dominum 》sur lequel je me suis souvent penché depuis une trentaine d'années.

En ne s'écartant pas des méthodes classiques, l'auteur propose pour les futaies régulières une formule simplifiée pour calculer la perte résultant d'une exploitation anticipée. C'est la formule qui convient aux peupleraies notamment, dans lesquelles il n'y a généralement pas de produits intermédiaires (d'éclaircies) entre la plantation et la réalisation en bloc du peuplement équienne, mais où il faut investir des frais de premier établissement: $r$.

Cette formule a été publiée une première fois, sous deux autres formes explicites dans la Revue des Eaux et Forêts de février 1944 (pages 84 et 85). En adoptant les données de M. Koebelé, ces formes sont les suivantes:

$$
1-\quad \mathrm{A}=(\mathrm{R}-\mathrm{r}) \frac{1, \mathrm{ot}^{\mathrm{m}}-1}{1, \mathrm{ot}^{\mathrm{n}}-1}+\mathrm{r}
$$

Cette première présentation de la formule offre l'intérêt de mettre en évidence l'erreur par excès (à l'avantage du propriétaire) commise par ceux qui ajoutent les frais de reboisement (r) à l'indemnité donnée par la formule simplifiée de la superficie, applicable aux peuplements se régénérant naturellement après exploitation en bloc.

$$
2-\quad \mathrm{A}=\mathrm{R} \frac{1, \mathrm{ot}^{\mathrm{m}}-1}{1, \mathrm{ot}^{\mathrm{n}}-1}+\mathrm{r}\left(1-\frac{1, \mathrm{ot}^{\mathrm{m}}-1}{1, \mathrm{ot}^{\mathrm{n}}-1}\right)
$$

Cette deuxième présentation, très voisine de celle proposée par l'auteur, a l'avantage de faire apparaître lấ grandeur de l'erreur commise par ceux qui se servent de la formule simplifiée de la superficie pour estimer la valeur relative des bois en croissance d'un peuplement d'origine artificielle.

Le dernier terme de la formule (2) représente « l'indemnité de reboisement $\gg q u$ 'il faut verser au propriétaire lésé ou dépossédé, en plus de la valeur d'avenir.

Cinq ans plus tard, Léon Schaefrer, dans son ouvrage " Principes d'Estimation forestière $\gg\left(1^{\text {re }}\right.$ édit., p. 143 et $2^{\circ}$ édit., p. 145) utilisait cette formule, que M. KoEBELÉ a eu le mérite de retrouver récemment par des voies différentes, en la présentant sous une troisième forme.

Pour tenir compte de toutes les éclaircies, l'auteur de l'article a eu recours à la méthode dite de la «valeur d'attente 》 basée sur l'escompte. C'est un procédé qui conduit à l'application, fractionnée ou non, de la formule 9 
de L. Schaeffer (op. cit.) Toutefois, il est important de remarquer que M. KoebelÉ désigne par 《valeur d'attente 》 la somme des valeurs actualisées des produits qui auraient été réalisés après la dépossession, tandis que L. SchaEfFer identifie la valeur d'attente à la valeur d'avenir et en fait l'inconnue. Cette remarque mérite d'être soulignée afin d'éviter toute confusion.

Il est à noter que la formule combinée, basée sur la capitalisation (prix de revient), portant le $\mathrm{n}^{\circ} 13$ dans le traité de L. SchaEFFER, donne aussi facilement le même résultat si le taux est correctement calculé, comme l'a fait M. Koebelé, avec aisance et bon sens. Pour l'exemple choisi $(\mathrm{m}=50$ ans): $\mathrm{A}=12395 \mathrm{NF}$ au lieu de $12401 \mathrm{NF}$; le faible écart étant dû aux décimales des tarifs d'I.C. La quantité entre parenthèses de la formule 13 - multipliée par «Tarif III 》 - est calculée une fois pour toutes. Aussi, cette formule permet-elle d'établir rapidement le tableau IV de l'auteur, pièce maîtresse de son article. D'autre part, son principal avantage théorique est de conduire, sans calcul, par simple suppression des termes qui n'ont pas de raison d'être, aux deux formules simplifiées de la superficie, qui ne sont que des cas particuliers, savoir: celle relative aux peuplements naturels du type taillis et celle, proposée par l'auteur, concernant normalement les peunlements d'origine artificielle du type peupleraie.

Ces considérations n'enlèvent rien à l'originalité de l'étude de $M$. Koebelé, qui a pour but de préconiser l'application d'une formule simplifiée aux futaies régulières, en compensant l'incidence financière due aux éclaircies par une réduction du taux réel de placement forestier. L'auteur a montré clairement, par des calculs comparatifs et des graphiques, que dans les futaies régulières d'épicéa de révolution moyenne, il suffisait de diminuer le taux de $40 \%$ environ pour obtenir une approximation suffisante. Tout reste à faire pour les autres futaies régulières, comme celles de pin, de sapin, de hêtre, de chêne, etc... Mais une voie est tracée. Peut-être sera-t-elle suivie.

V.-R. VINÇONNEAU.

\section{Les sondages à la tarière en Roumanie}

A la suite d'un article paru dans la Revue Forestière Française dans le numéro de juin 1958 (《 Tarière de Pressler sans temps de passage 》), nous avons reçu de M. l'Ingénieur R. DIsSESCU, de l'Institut roumain de Recherches forestières, la lettre suivante dont nous publions les extraits susceptibles, croyons-nous, d'intéresser les lecteurs:

«Nous avons lu dans la Revue Forestière Française, l'article intitulé «Tarière de Pressler sans temps de passage ».

«Dans cet article, il y a un paragraphe concernant les modalités de collection et de transport des barrettes de sondage, extraites avec la tarière de Pressler.

«Récemment, notre collègue Minnea Stäanescu s'est occupé lui aussi de ce problème, et $\mathrm{je}$ vais vous présenter en quelques mots le système qu'il emploie.

« Il s'agit d'une boite légère parallélépipédique, en bois ou en plastique, de dimensions variables selon les nécessités, dans laquelle on introduit un compartimentage cellulaire construit en carton mince rigide.

« Sur les bords de la boîte, on marque chaque rang et chaque colonne avec lettres et chiffres comme la notation d'un échiquier. I1 est ainsi facile d'identifier une carotte de sondage mise en boîte par un indice contenant le numéro caractéristique de la boîte (en chiffres romains), le numéro caractéristique de la colonne (en chiffres arabes) et la lettre indiquant le rang.

« Comme dans une boite de $12 \times 12 \mathrm{~cm}$ (dimensions intérieures), on peut placer facilement 225 carottes de sondage, une telle boite est suffisante pour 
une journée de travail. Elle peut naturellement être employée un temps presque indéfini. 》

Les lecteurs trouveront ci-après le dessin de la boite en cause, comme nous l'a également communiqué l'auteur de la lettre.

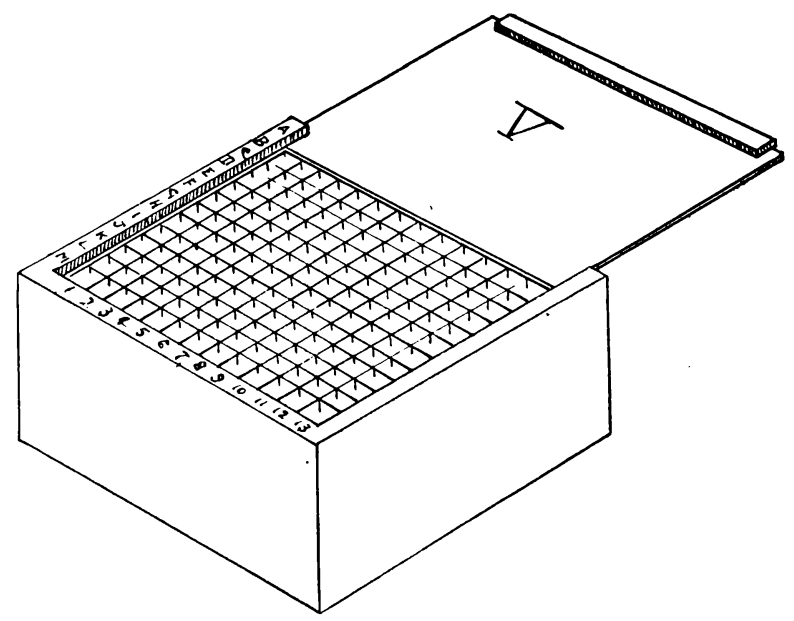

Boite pour « carottes » de sondage. 